\title{
AtHAP3b Plays a Crucial Role in the Regulation of Flowering Time in Arabidopsis during Osmotic Stress
}

\author{
Nai-Zhi Chen, Xiu-Qing Zhang, Peng-Cheng Wei, Qi-Jun Chen, Fei Ren, Jia Chen and Xue-Chen Wang* \\ State Key Laboratory of Plant Physiology and Biochemistry, College of Biological Sciences, China Agricultural University, \\ Beijing 100094, China
}

Received 21 March 2007, Accepted 1 June 2007

The HAP complex has been found in many eukaryotic organisms. HAP recognizes the CCAAT box present in the promoters of $30 \%$ of all eukaryotic genes. The HAP complex consists of three subunits - HAP2, HAP3 and HAP5. In this paper, we report the biological function of the $A t H A P 3 b$ gene that encodes one of the HAP3 subunits in Arabidopsis. Compared with wild-type plants, hap3b-1 and hap3b-2 mutants exhibited a delayed flowering time under long-day photoperiod conditions. Moreover, the transcription levels of $F T$ were substantially lower in the mutants than in the wild-type plants. These results imply that $A t H A P 3 b$ may function in the control of flowering time by regulating the expression of $F T$ in Arabidopsis. In a subsequent study, $A t H A P 3 b$ was found to be induced by osmotic stress. Under osmotic stress conditions, the hap $3 b$ 1 and hap3b-2 mutants flowered considerably later than the wild-type plants. These results suggest that the $A t H A P 3 b$ gene plays more important roles in the control of flowering under osmotic stress in Arabidopsis.

Keywords: Arabidopsis, AtHAP3b, Flowering time, HAP complex, Osmotic stress

\section{Introduction}

The CCAAT box is one of the most common elements in eukaryotic promoters. It is present in $30 \%$ of all eukaryotic genes and is located $80-100 \mathrm{bp}$ upstream of the transcription start site (Bucher, 1990; Maity and de Crombrugghe, 1998; Mantovani, 1999). The HAP complex (also known as CBF, NF-Y, or CP1) was isolated as a CCAAT-binding protein complex, and it is an evolutionarily conserved transcription

\footnotetext{
*To whom correspondence should be addressed.

Tel: 86-10-62732706; Fax: 86-10-62733450

E-mail: xcwang@cau.edu.cn
}

factor that occurs in a wide range of organisms from yeast to humans. It includes three subunits: NF-YA (CBF-B or HAP2), NF-YB (CBF-A or HAP3), and NF-YC (CBF-C or HAP5). They are all required for DNA binding (Mantovani, 1999). A fourth subunit, HAP4 is present in yeast; it does not bind to DNA but is required for complex formation (Forsburg and Guarente, 1989; McNabb et al., 1997). In yeast and humans, each subunit of the HAP complex is encoded by a single gene, whereas in plants, each subunit of the HAP complex is encoded by multiple genes. In Arabidopsis, there are 10, 10 and 9 genes encoding the HAP2, HAP3 and HAP5 subunits, respectively. Further, 11 putative HAP3 genes and 13 putative HAP5 genes have been recognized in rice (Edwards et al., 1998; Gusmaroli et al., 2001; Gusmaroli et al., 2002; Miyoshi et al., 2003).

In recent years, several HAP subunits have been identified in plants. The first was isolated from maize as a HAP3 homolog (Li et al., 1992). The Bn CBF-B subunit was identified in Brassica napus as a plant homolog of HAP2 (Albani and Robert, 1995). MtHAP2-1 in the model legume Medicago truncatula plays a key role in nodule development (Combier et al., 2006). There are 3 OsHAP3 subunits (OsHAP3A, OsHAP3B, and OsHAP3C), and they function during chloroplast development (Miyoshi et al., 2003). The Arabidopsis HAP3 subunits LEC1 and LEC1-LIKE (L1L) play important roles in embryogenesis (Lotan et al,. 1998; Kwong et al., 2003; Lee et al., 2003).

Interestingly, the HAP subunits can function not only by complex formation but also by individual interaction with other proteins. It was reported that the AtHAP3 and AtHAP5 subunits can interact with the CCT domain of the CONSTANS (CO), CONSTANS-LIKE, and TOC1 proteins (Wenkel et al., 2006). HAP2 contains two important domains: NF-YA1 interacts with the HAP3/HAP5 dimer during the formation of the trimeric HAP complex, while NF-YA2 interacts with the CCAAT box. The CCT domain is highly similar to the NFYA2 domain in terms of structure (Xing et al., 1994; Robson et al., 2001; Romier et al., 2003). Recent reports have revealed 
that the $\mathrm{CCT}$ domain protein $\mathrm{CO}$ forms a heterotrimeric $\mathrm{CO} /$ AtHAP3/AtHAP5 complex by interacting with the AtHAP3/ AtHAP5 dimer, similar to the AtHAP2 subunit. CO functions in the photoperiodic flowering pathway. Consequently, the heterotrimeric CO/AtHAP3/AtHAP5 complex also plays an important role in this pathway. Overexpression of the AtHAP3a and AtHAP2a subunits in Arabidopsis has been shown to delay flowering (Wenkel et al., 2006).

In this study, we investigated the biological function of AtHAP3b in Arabidopsis. We examined the expression pattern of $A t H A P 3 b$ and obtained two AtHAP $3 b$ T-DNA insertion mutants. Our result showed that the hap $3 b-1$ and hap $3 b-2$ mutants flowered later than wild-type plants under long-day photoperiod conditions. In addition, we found that $A t H A P 3 b$ was induced by osmotic stress. Further, the difference of flowering time between the hap $3 b$ mutants and wild-type plants was substantially higher under osmotic stress conditions. In conclusion, our results indicated that $A t H A P 3 b$ is required for the regulation of flowering time under osmotic stress conditions.

\section{Materials and Methods}

Plant materials and growth conditions. Arabidopsis thaliana ecotype Columbia seeds were surface-sterilized in a solution of $0.5 \% \mathrm{NaClO}$ for $15 \mathrm{~min}$, washed five times with sterile distilled water and stratified at $4^{\circ} \mathrm{C}$ for $2 \mathrm{~d}$ in the dark. Following this treatment, the plants were germinated and grown on Murashige and Skoog medium, supplemented with $3 \%(\mathrm{w} / \mathrm{v})$ sucrose and $0.8 \%$ $(\mathrm{w} / \mathrm{v})$ agar, $\mathrm{pH}$ 5.7. Germination was executed in a growth chamber under long-day photoperiod conditions (16-h light/8-h dark cycle) at $22^{\circ} \mathrm{C}$.

Histochemical analysis of GUS expression. To examine the tissue-specific expression of $A t H A P 3 b, 1066 \mathrm{bp}$ of the $A t H A P 3 b$ promoter region was amplified using 5'-CTGCAGTGGTTCGCCT ACTCTTTCA-3' and 5'-GAATTCAGTGGTGAAGGAGTCGCCG$3^{\prime}$ (enzyme digestion site underlined) primer sequences. The amplified product was cloned into the the PstI and EcoRI sites of pCAMBIA1391 GUS activity assays were performed as follows: transgenic plants were incubated in 5-bromo-4-chloro-3-indolyl glucuronide (X-gluc) buffer [100 mM sodium phosphate buffer $\mathrm{pH}$ 7.0, $0.1 \mathrm{mM}$ EDTA, $0.5 \mathrm{mM} \mathrm{K}_{3} \mathrm{Fe}(\mathrm{CN})_{6}, 0.5 \mathrm{mM} \mathrm{K}_{4} \mathrm{Fe}(\mathrm{CN})_{6}, 1 \mathrm{mM}$ $\mathrm{X}$-gluc and $0.1 \%$ Triton $\mathrm{X}-100$ ] at $37^{\circ} \mathrm{C}$ for $12 \mathrm{~h}$; seedlings were washed to clear chlorophyll with $70 \%$ ethanol. For GUS expression analysis under various stress conditions, 10-day-old AtHAP3b::GUS transgenic plants grown on MS medium were treated by various stresses and then incubated in X-gluc buffer at $37^{\circ} \mathrm{C}$ for $1 \mathrm{~h}$ or $3 \mathrm{~h}$.

RT-PCR and quantitative real-time RT-PCR analysis. Total RNA was extracted from 4-week-old seedlings with Trizol Reagent (Invitrogen). Reverse transcription reactions were performed using the M-MLV Reverse Transcriptase (Promega) with the oligo $(\mathrm{dT})_{18}$ primers. The AtHAP $3 b$ gene was amplified using gene-specific primers 5'-TCTTCCCACTCAAGTCAACC-3' and 5'-CACCTCC ACTGTCGCTACC-3'. The ACTIN2/8 mRNA expression was amplified as an internal control using the specific primers 5'TCTTCCGCTCTTTCTTTCCA-3' and 5'-GAGAGAACAGCTTG GATGGC-3'.

Quantitative real-time RT-PCR was performed as described (Chai et al., 2006) with specific primers of AtHAP3b gene 5'AGTGTGTCTCCGAGTTCAT-3' and 5'-TAGTCATAGCCCAGAG CAAA-3'. And quantitative real-time RT-PCR analysis of $F T$ and $A P 1$ was performed using the specific primers of $F T$ gene 5'CGCCAGAACTTCAACACTCG-3' and 5'-TCTTCCTCCGCAG CCACT-3', or the specific primers of $A P 1$ gene 5'-ACAATATGCC TCCCCCTC-3' and 5'-CTTCTTGATACAGACCACCC-3'. The ACTIN2/8 mRNA expression was amplified as an internal control for real-time RT-PCR analysis using the specific primers 5'GGTAACATTGTGCTCAGTGGTGG-3' and 5'-CACGACCTTAAT CTTCATGCTGC-3'. And quantitative PCR experiments were repeated three independent times.

Identification of the T-DNA insertion mutants for the $\boldsymbol{A t H A P B}$ gene. T-DNA insertion lines of the AtHAP3b gene (At5g47640), named as hap3b-1 (SALK_061365) and hap3b-2 (SALK_025666) (http://signal.salk.edu) were obtained from NASC. The $\bar{A} t H A P 3 b$ homozygous mutants were screened by PCR amplification using the following primers. hap $3 b-1$ LP: 5'-CAATGGTTGGACCCAC AGGAG-3', RP: 5'-CCTGCATCGTCTCTTTGGCAT-3', hap3b-2 LP: 5'-GACGGTCGAGCACGTATCCAG-3', RP: 5'-TGGGTCGT AAAAATAGGCATGTG-3', and the primer from T-DNA region LBb1: 5'-GCGTGGAAAAACCGCTTGCTGCAACT-3'. The expression of the $A t H A P 3 b$ gene in hap $3 b-1$ and hap $3 b-2$ mutant plants was examined by reverse transcript PCR analysis.

Complementation of the T-DNA insertional mutants hap $3 b-1$ and hap3b-2. The AtHAP3 genomic region was amplified using $P f u$ DNA polymerase from Arabidopsis genomic DNA with the primers 5'-CTGCAGGAGGTTTGTTTGTTTTTCTTTG-3' and 5'GGATCCCTTTGCTGCCTCTCTATGTA-3' (enzyme digestion site underlined). The amplified regions were cloned into the Pst I/ BamHI sites of pCAMBIA1391. The construct was introduced into Agrobacterium strain GV3101 and transformed into hap $3 b-1$ and hap $3 b-2$ mutants.

Measurement of flowering time. The flowering time was measured as total leaf number as described previously (Koornneef et al., 1998). The total leaf number was recorded including the number of rosette leaves and the number of cauline leaves on the main stem when the first flower opened.

Analysis of the flowering time phenotype under osmotic stress conditions. For quantitative real-time RT-PCR analysis, 2-week-old seedlings grown on MS medium were immersed in a solution containing $300 \mathrm{mM}$ mannitol for 1, 3, 6, 12 and $24 \mathrm{~h}$, with distilled water as a control. To analyze the effect of osmotic stress on the flowering time phenotype, 10-day-old seedlings grown on MS medium were transferred to MS medium containing $100 \mathrm{mM}$ mannitol. The plants were photographed after 49 days of growth. 


\section{Results and Discussion}

Expression pattern of $A t H A P 3 b$ in different tissues and at different developmental stages. To determine the expression of $A t H A P 3 b$ in various tissues, we constructed the $A t H A P 3 b$ promoter::GUS fusion gene to transform wild-type Arabidopsis plants. The GUS signal was observed in almost the entire 10day-old seedling, although the roots exhibited a weaker signal (Fig. 1(a) A) and the fusion gene was not expressed at all in the root tip (Fig. 1(a) F). However, in mature seedlings, the GUS activity in the roots was reduced and/or nearly absent (Fig. 1(a) G). A strong signal was observed in the rosette and cauline leaves, particularly the vascular tissues, of 4-week-old seedlings (Fig. 1(a) B). The GUS signal was absent in mature stems (Fig. (a) E) and present at both ends of mature siliques (Fig. 1(a) C). Among the floral organs, only calyces and filaments exhibited strong staining (Fig. 1(a) D).

To quantitatively examine the mRNA expression levels of AtHAP3b in various tissues, RNA was extracted from various tissues including the roots, stems, flowers and rosette and cauline leaves. We found that the flowers and cauline leaves exhibited the highest transcription level of $A t H A P 3 b$ (Fig. 1(b)). Previous studies also showed that most HAP subunits are expressed during flower development (Edwards et al., 1998; Gusmaroli et al., 2001; Gusmaroli et al., 2002).

AtHAP3b expression during the developmental stages was also examined by quantitative real-time RT-PCR analysis. RNA was extracted from 4-, 7-, 14-, 21- and 38-day-old seedlings. The results showed that the level of $A t H A P 3 b$ mRNA peaked in 38-day-old seedlings (Fig. 1(c)). At this stage, $30-50 \%$ of the flower buds had opened. In other words, the transcription level of $A t H A P 3 b$ during the flowering stage was higher than that during other stages.

\section{Expression pattern of the $A t H A P 3 b$ gene in response to} osmotic stress. The expression pattern of the $A t H A P 3 b$ gene in response to osmotic stress was analyzed using quantitative real-time RT-PCR. AtHAP3b expression was strongly induced by osmotic stress. The expression level of AtHAP $3 b$ peaked after $1 \mathrm{~h}$ of osmotic stress treatment (Fig. 2(a)). These results indicate that $A t H A P 3 b$ may be involved in the response to osmotic stress.

We further analyzed the pattern of GUS expression driven by the $A t H A P 3 b$ promoter under osmotic stress conditions in AtHAP3b::GUS transgenic plants. As expected, after 1-h incubation in X-gluc buffer at $37^{\circ} \mathrm{C}$, GUS staining revealed that the transcript levels of the GUS reporter gene were obviously increased under osmotic stress. Further, no GUS signal was observed in both transgenic Arabidopsis plants treated with distilled water and plants grown on MS medium (Fig. 2(b)). This result confirmed that AtHAP3b was induced by osmotic stress. After 3-h incubation in X-gluc buffer at $37^{\circ} \mathrm{C}$, GUS staining could be detected in all materials; however, the GUS signal observed in the transgenic plants grown on MS medium was much weaker than that in plants (a)
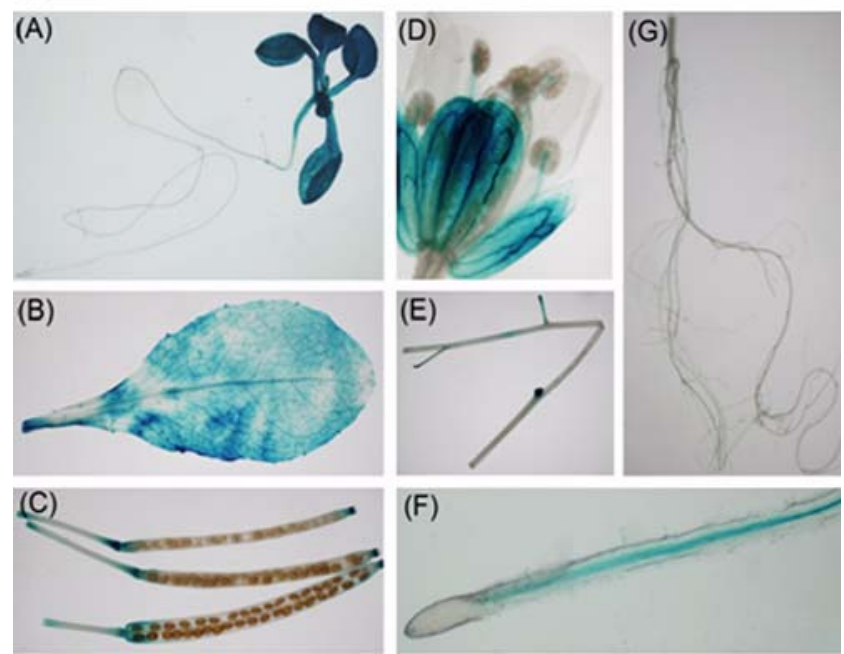

(b)

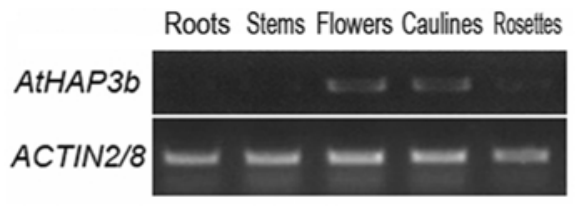

(c)

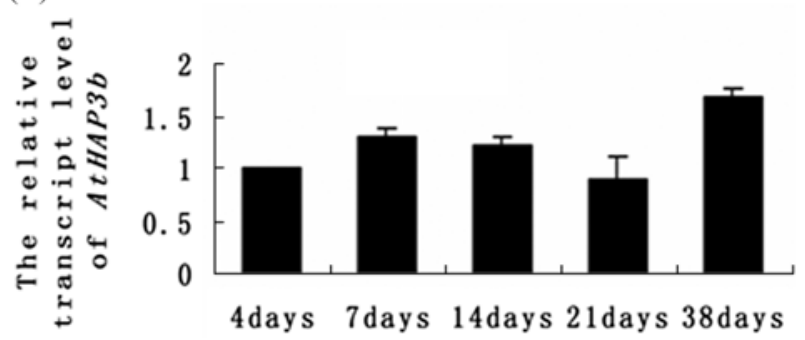

Fig. 1. Expression pattern of $A t H A P 3 b$ in various tissues and at different developmental stages. (a) Histochemical staining analysis of GUS activity in AtHAP $3 b$ promoter::GUS transgenic Arabidopsis plants. (A) 10-day-old seedling. (B) Rosette leaf of a 4-week-old plant. (C) Mature siliques. (D) Flower of a 4-week-old plant. (E) Stem of a 4-week-old plant. (G) Roots of a 4-week-old plant. (F) Root tip of a 10-day-old seedling. (b) RT-PCR analysis of $A t H A P 3 b$ transcripts in various tissues of Arabidopsis plants. Total RNA was extracted from various tissues including the roots, stems, flowers cauline and rosette leaves of wild-type plants grown in the growth chamber with a $16 \mathrm{~h}$ light $/ 8 \mathrm{~h}$ dark cycle at $22^{\circ} \mathrm{C}$. (c) $A t H A P 3 b$ expression patterns in different developmental stages. Total RNA was extracted from 4-, 7-, 14-, 21- and 38-day-old seedlings. Quantitative real-time RT-PCR was performed using $A t H A P 3 b$ specific primers.

grown under osmotic stress conditions. Compared with the transgenic plants grown on MS medium, those treated with distilled water also exhibited increased GUS expression (Fig. 2(c)). This result indicates that $A t H A P 3 b$ may also be induced by waterlogging stress. In summary, these data further confirmed the inducible expression of $A t H A P 3 b$ under stress conditions. Consistent with our results, it has been shown that 

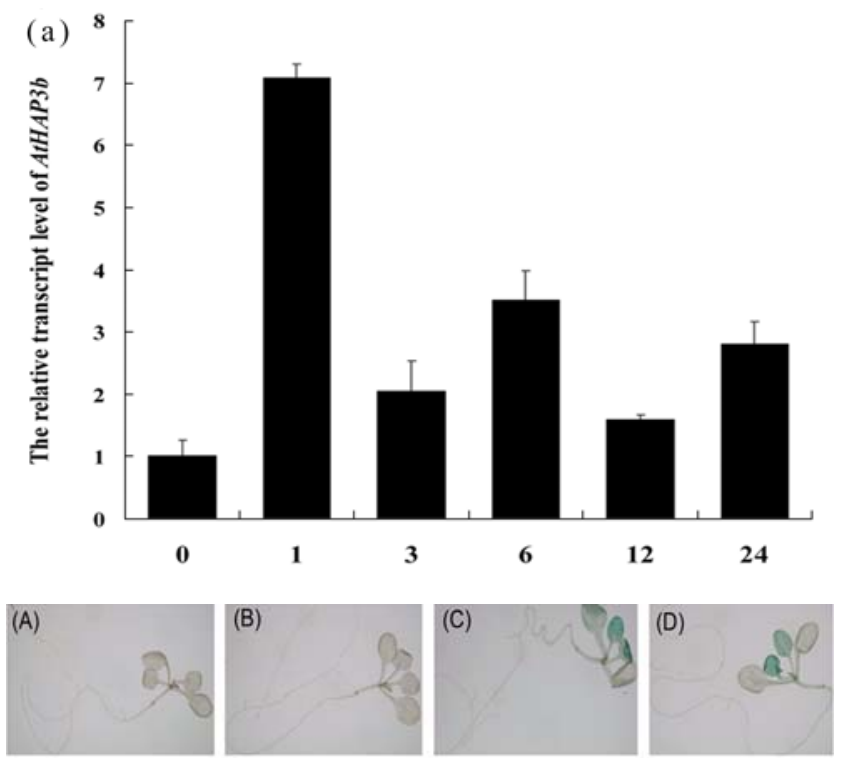

(b)
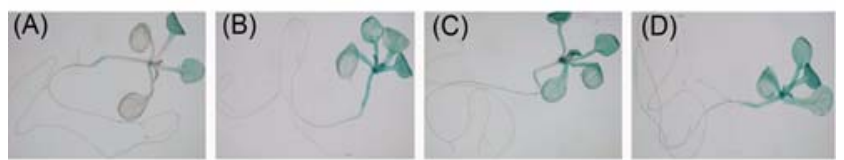

(c)

Fig. 2. Analysis of $A t H A P 3 b$ gene expression under osmotic stress conditions. (a) Quantitative real-time RT-PCR analysis of AtHAP3b gene expression under osmotic stress conditions. Twoweek-old wild-type plants were submerged in $300 \mathrm{mM}$ mannitol for $1,3,6,12$ and $24 \mathrm{~h}$. The treated plants were then frozen in liquid nitrogen, and total RNA was extracted for real-time PCR analysis. (b) Histochemical analysis of GUS activity in $A t H A P 3 b$ promoter::GUS transgenic Arabidopsis plants under osmotic stress conditions. Ten-day-old transgenic seedlings grown on MS medium were transferred to a solution containing 300 or $400 \mathrm{mM}$ mannitol or distilled water for $1 \mathrm{~h}$. The plants were then immersed for $1 \mathrm{~h}$ in $\mathrm{X}$-gluc buffer at $37^{\circ} \mathrm{C}$. (A) MS medium control. (B) Distilled water $\left(\mathrm{H}_{2} \mathrm{O}\right)$ treatment. (C) Treatment with $300 \mathrm{mM}$ mannitol. (D) Treatment with $400 \mathrm{mM}$ mannitol. (c) Histochemical analysis of GUS activity in AtHAP3b promoter::GUS transgenic Arabidopsis plants under osmotic stress conditions. The transgenic plants were immersed in X-gluc buffer for $3 \mathrm{~h}$ at $37^{\circ} \mathrm{C}$. (A) MS medium control. (B) Distilled water $\left(\mathrm{H}_{2} \mathrm{O}\right)$ treatment. (C) Treatment with $300 \mathrm{mM}$ mannitol. (D) Treatment with $400 \mathrm{mM}$ mannitol.

AtHAP3b expression is not detectable in liquid-cultured plants, while high expression is observed in soil-grown plants; this data also suggested the possible role of $A t H A P 3 b$ in regulation of the osmotic stress response (Edwards et al., 1998).

Identification and complementation of the T-DNA insertion mutants hap3b-1 and hap3b-2. To examine the function of the $A t H A P 3 b$ gene in plants, two mutants with a TDNA insertion in the $A t H A P 3 b$ genomic sequence were
(A)

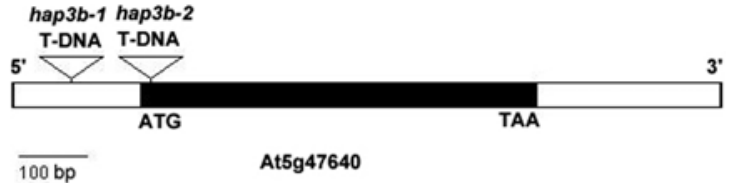

(B)

(C)

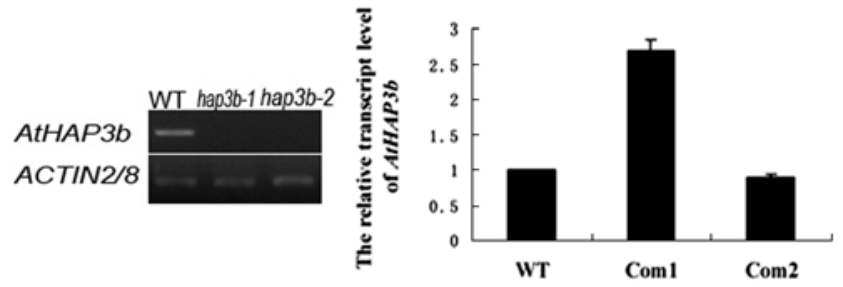

Fig. 3. Analysis of AtHAPBb expression levels in wild-type, mutant and complementation line plants. (A) Illustration of the T-DNA insertions in $A t H A P 3 b$. The black box indicates the exon and the white boxes, untranslated regions. Triangles indicate the positions of T-DNA insertion. This illustration is drawn to scale. (B) RT-PCR analysis of $A t H A P 3 b$ transcripts in wild-type plants and T-DNA insertion mutant lines hap $3 b-1$ and hap $3 b-2$. Total RNA was isolated from 4-week-old whole plants grown under normal conditions. RT-PCR was performed with $A t H A P 3 b$-specific primers, and $A C T I N 2 / 8$ expression levels were analyzed as an internal control. (C) Analysis of the transcript levels of the $A t H A P 3 b$ gene in the wild-type and complementation line plants. WT: wild-type plants, Com1: complementation line of hap $3 b-1$ plants, Com2: complementation line of hap $3 b-2$ plants.

obtained. In the hap $3 b-1$ mutant, the T-DNA was inserted in the 5-untranslated region and in the hap $3 b-2$ mutant, it was inserted in the coding region (Fig. 3(A)). RT-PCR analysis showed that $A t H A P 3 b$ mRNA was not detectable in the hap $3 b-1$ and hap3b-2 mutants (Fig. 3(B)). This result indicated that $A t H A P 3 b$ expression was lost in hap $3 b-1$ and hap $3 b-2$ plants. Two homozygous lines of hap $3 b-1$ and hap $3 b-2$ were used for further analysis.

The two homozygous lines Com1 and Com2 were selected as complementation lines of hap $3 b-1$ and hap $3 b-2$ plants respectively. Quantitative real-time RT-PCR analysis revealed that the transcript levels of $A t H A P 3 b$ in the Com 1 plants were slightly higher than those in wild-type Arabidopsis plants. However, the transcript levels of $A t H A P 3 b$ in the Com 2 plants were similar to those in wild-type Arabidopsis plants (Fig. 3(C)).

Flowering time phenotypes of hap3b-1 and hap3b-2 mutants and their complementation lines. The observed AtHAP $3 b$ expression patterns suggest that AtHAP3b may function in flowering control. To further determine the effect of $A t H A P 3 b$ on flowering time, we observed the phenotype of hap $3 b-1$ and hap3b-2 mutants and wild-type plants under long-day photoperiod conditions. We found that under these conditions, the two AtHAP3b-knockout mutants hap $3 b-1$ and hap $3 b-2$ flowered later than wild-type plants. Furthermore, the flowering time phenotypes of hap $3 b-1$ and hap $3 b-2$ could 


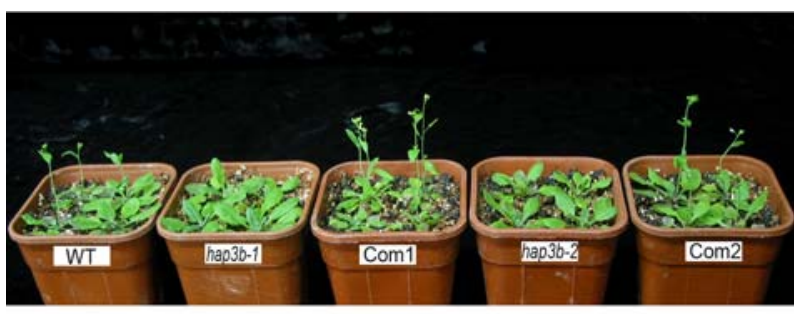

(A)

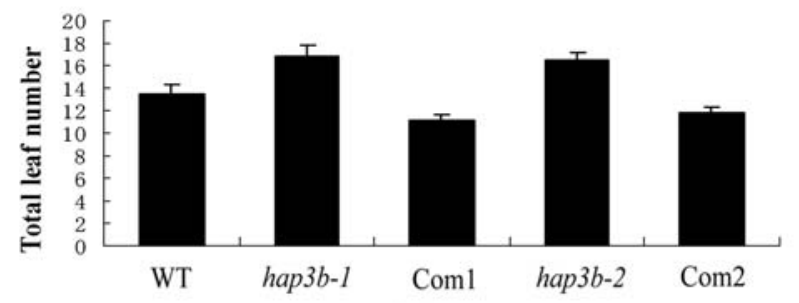

(B)

Fig. 4. Flowering phenotypes of 30-day-old AtHAP3b T-DNA insertion mutants and their complementation lines under long-day photoperiod conditions. (A) Flowering phenotypes of the hap $3 b-1$ and hap $3 b-2$ mutants and their complementation lines. (B) Total number of leaves of the hap $3 b-1$ and hap $3 b-2$ mutants and their complementation lines. WT: wild-type plants, hap $3 b-1$ and hap $3 b-$ 2: AtHAP3b T-DNA insertion lines, Com1 and Com2: complementation lines of hap $3 b-1$ and hap $3 b-2$, respectively.

be rescued by complementation of $A t H A P 3 b$. Under long-day photoperiod conditions, plants of the Com1 and Com2 exhibited similar flowering time phenotypes, although slightly earlier than wild-type plants (Fig. 4(A)). Flowering time also is indicated by the total number of leaves (Fig. 4(B)).

To further confirm the above results, we analyzed the expression patterns of several genes related to flowering control by real-time RT-PCR in hap $3 b-1$ and hap $3 b-2$ mutants and their respective complementation lines (Fig. 5). The transcription levels of $F T$ and $A P 1$ were considerably lower in the mutants than those in the wild-type plants (Fig. 5). As expected, the expression levels of these genes in the complementation lines were dramatically higher than that in the hap $3 b$ mutants. Moreover, the FT and APl transcripts were more abundant in the complementation lines than in the wild-type plants. However, the expression levels of $F L C, C O$, $F V E$, and GI did not obviously differ between the mutant and wild-type plants. Additionally, it is known that $A P 1$ is an important gene for floral transition and that it acts downstream of FT (Ruiz-García et al., 1997; Wigge et al., 2005). Taken together, these results suggest that $A t H A P 3 b$ plays an important role in flowering control by regulating $F T$ expression.

However, unlike the AtHAP $3 b$ mutation, the AtHAP $3 a$ mutation did not cause a change in the flowering time from that in the wild-type (Wenkel et al., 2006). AtHAP3b may be an important factor distinct from AtHAP3a. Different HAP genes probably have different functions in the control of flowering time.
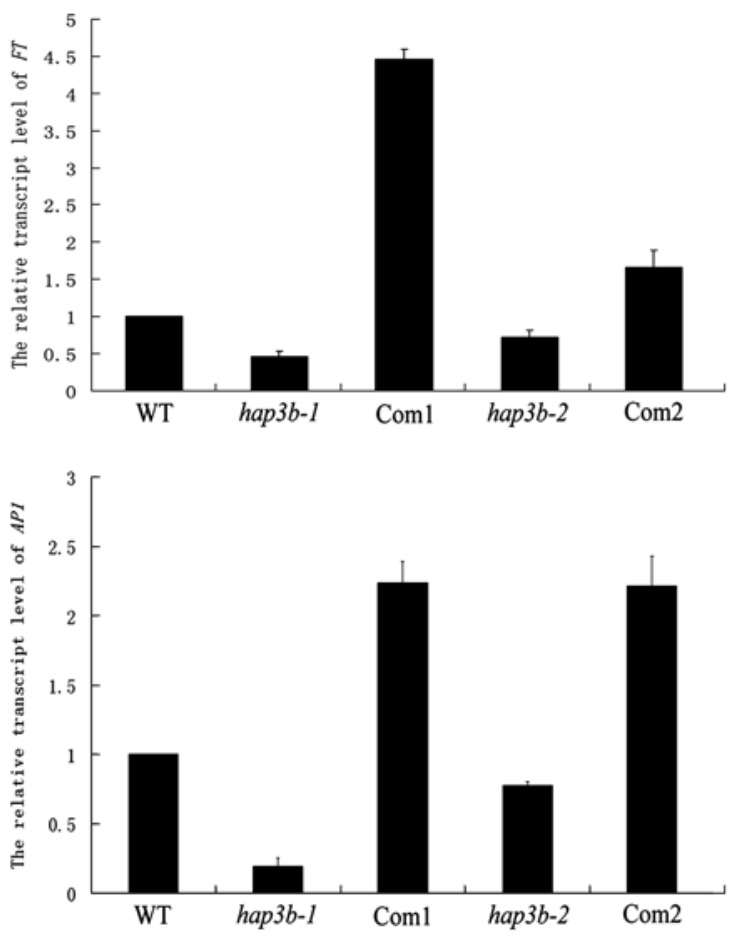

Fig. 5. Quantitative real-time RT-PCR analysis of $F T$ and $A P I$ expression levels in the mutants and their complementation lines. Total RNA was extracted from 24-day-old seedlings of WT, hap $3 b$ mutants, or the complementation lines under long-day photoperiod conditions. The $A C T I N 2 / 8$ gene was used as the quantitative control. The experiment was repeated three times. WT: wild-type plants, hap $3 b-1$ and hap $3 b-2:$ AtHAP3b T-DNA insertion mutants, Com1 and Com2: complementation lines of hap $3 b-1$ and hap $3 b-2$, respectively.

Effect of osmotic stress on the phenotype of hap $3 b-1$ and hap3b-2 mutants and their complementation lines. As observed from the above results, AtHAP $3 b$ was strongly induced by osmotic stress (Fig. 2(a)), and under normal growth conditions, its knockout mutants flowered later than wild-type plants (Fig. 4(A)). To further examine the function of the AtHAP3b gene under osmotic stress conditions, we assessed the osmotic stress tolerance. 6-day-old seedlings were transferred to MS plates containing 350, 400, and 450 $\mathrm{mM}$ mannitol. Next, the plates were placed in the vertical position, and the root tips were oriented upward for 9 days. The AtHAP3b-knockout mutants hap $3 b-1$ and hap $3 b-2$ were very slightly sensitive to osmotic stress compared to the wildtype plants (data not shown). This result suggests that AtHAP $3 b$ may be not necessary for the response to severe osmotic stress.

To further examine the effect of osmotic stress on the flowering time phenotype, 10-day-old seedlings of hap $3 b-1$, hap $3 b-2$, the two complementation lines Com 1 and Com2, and wild-type plants were transferred to MS medium containing $100 \mathrm{mM}$ mannitol. After 33 days of growth under osmotic stress conditions, most of the wild-type plants flowered, but 

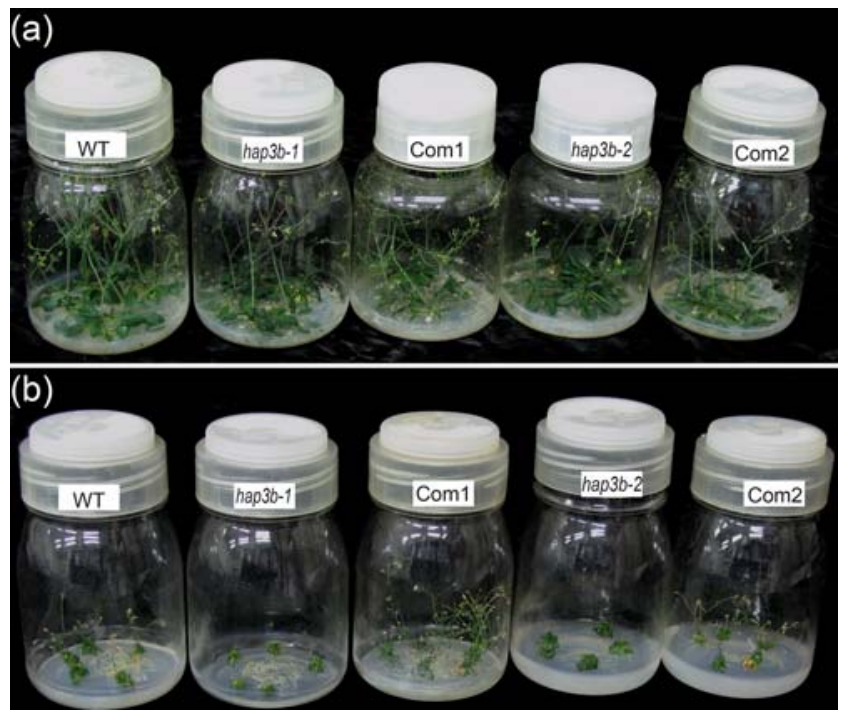

Fig. 6. Effect of osmotic stress on the flowering time phenotypes in hap $3 b-1$ and hap $3 b-2$ mutants and their complementation lines under long-day photoperiod conditions. (a) MS medium control. (b) Treatment with $100 \mathrm{mM}$ mannitol. WT: wild-type plants, hap $3 b-1$ and hap3b-2: AtHAP3b T-DNA insertion lines, Com1 and Com2: complementation lines of hap $3 b-1$ and hap $3 b-2$, respectively.

the hap $3 b-1$ and hap $3 b-2$ plants did not. Further, all hap $3 b-1$ and hap $3 b-2$ plants grown on MS medium as a control flowered. However, under osmotic stress conditions, the hap $3 b-1$ and hap $3 b-2$ plants did not begin to flower even after 49 days of growth, while all the wild-type plants flowered (Fig. 6). On the other hand, in plants of the two complementation lines Com 1 and Com2, a similar flowering time phenotype to that of the wild-type plants was observed under osmotic stress conditions. As mentioned above, our results suggest that the main function of $A t H A P 3 b$ is perhaps the regulation of flowering time under osmotic stress conditions. It is known that there may be a genetic link between cold stress response and flowering time involving the FVE and GI genes in Arabidopsis (Kim et al., 2004; Cao et al., 2005). Intermittent cold treatments delayed flowering in wild-type plants but not in fve mutants. When gi-3 plants were subjected to intermittent cold treatments, their flowering time was delayed more than that of wild-type plants. Similarly, AtHAP $3 b$ may be a genetic link between the flowering time and osmotic stress response in Arabidopsis. However, the precise molecular mechanism of $A t H A P 3 b$ in the regulation of flowering time under osmotic stress conditions remains unclear. Further analysis would provide novel information to help us understand the function of AtHAP3b.

In summary, one important conclusion of our experiments was that $A t H A P 3 b$ may play an important role in flowering control by regulating the expression of FT under osmotic stress conditions. Our data provide useful information for future studies regarding flowering control and the osmotic stress response facilitated by HAP subunits. Further research is required to identify the precise molecular mechanism by which $A t H A P 3 b$ regulates flowering time.

Acknowledgments We thank the Arabidopsis Biological Research Center for kindly providing the T-DNA insertional mutant seeds. This work was supported by grants from the National Basic Research Program of China (No. 2006CB100100 and No. 2003CB114300) and the National Natural Science Foundation of China (No. 30370129 and No.30421002)

\section{References}

Albani, D. and Robert, L. S. (1995) Cloning and characterization of a Brassica napus gene encoding a homologue of the Bsubunit of a heteromeric CCAAT-binding factor. Gene 167, 209-213.

Bucher P., (1990) Weight matrix descriptions of four eukaryotic RNA polymerase II promoter elements derived from 502 unrelated prolaboratory. J. Mol. Biol. 212, 563-578.

Cao, S. Q., Ye, M. and Jiang, S. T. (2005) Involvement of GIGANTEA gene in the regulation of the cold stress response in Arabidopsis. Plant Cell Rep. 24, 683-690.

Chai, M. F., Wei, P. C., Chen, Q. J., An, R., Chen, J., Yang, S. and Wang, X. C. (2006) NADK3, a novel cytoplasmic source of NADPH, is required under conditions of oxidative stress and modulates abscisic acid responses in Arabidopsis. Plant $J$. 47, 665-674.

Combier, J. P., Frugier, F., de Billy, F., A. Boualem, El-Yahyaoui, F., Moreau, S., Vernié, T., Ott, T., Gamas, P., Crespi, M. and Niebel, A. (2006) MtHAP2-1 is a key transcriptional regulator of symbiotic nodule development regulated by microRNA169 in Medicago truncatula. Genes Dev. 20, 3084-3088.

Edwards, D., Murrey, J. A. H. and Smith, A. G., (1998) Multiple genes coding the conserved CCAAT-box transcription complex are expressed in Arabidopsis. Plant Physiol. 117, 1015-1022.

Forsburg, S. L. and Guarente, L., (1989) Identification and characterization of HAP4: a third component of CCAATbinding HAP2/HAP3 heteromer. Genes Dev. 3, 1166-1178.

Gusmaroli, G., Tonelli, C., and Mantovani, R. (2002) Regulation of novel members of the Arabidopsis thaliana CCAAT-binding nuclearfactor Y subunits. Gene 283, 41-48.

Gusmaroli, G., Tonelli, C. and Mantovani, R., (2001) Regulation of the CCAAT-binding NF-Y subunits in Arabidopsis thaliana. Gene 264, 173-185.

Kim, H. J., Hyun, Y., Park, J. Y., Park, M. J., Park, M. Y., Kim, M. D., Kim, H. J., Lee, M. H., Moon, J., Lee, I. and Kim J. (2004) A genetic link between cold responses and flowering time through FVE in Arabidopsis thaliana. Nat. Genet. 36, 167-171.

Koornneef, M., Alonso-Blanco, C., Blankestijn-de Vries, H., Hanhart, C. and Peeters, A. (1998) Genetic interactions among late-flowering mutants of Arabidopsis. Genetics 148, 885-892.

Kwong, R. W., Bui, A. Q., Lee, H., Kwong, L. W., Fischer, R. L., Goldberg, R. B. and Harada, J. J. (2003) LEAFY COTYLEDON1-LIKE defines a class of regulators essential for embryo development. Plant Cell 15, 5-18.

Lee, H. S., Fischer, R. L., Goldberg, R. B. and Harada, J. J. (2003) Arabidopsis LEAFY COTYLEDON1 represents a 
functionally specializedsubunit of the CCAAT binding transcription factor. Proc. Natl. Acad. Sci. USA 100, 2152-2156.

Li, X. Y., Mantovani, R., Vanhuijsduijnen, R. H., Andre, I., Benoist, C. and Mathis, D. (1992) Evolutionary variation of the CCAAT-binding transcription factor NF-Y. Nucleic Acids Res. 20, 1087-1091.

Lotan, T., Ohto, M., Yee, K. M., West, M. A., Lo, R., Kwong, R. W., Yamagishi, K., Fischer, R. L., Goldberg, R. B. and Harada, J. J. (1998) Arabidopsis LEAFY COTYLEDON1 is sufficient to induce embryo development in vegetative cells. Cell 93, 1195-1205.

Maity, S. and de Crombrugghe, B. (1998) Role of the CCAATbinding protein CBF/NF-Y in transcription. Trends Biochem. Sci. 23, 174-178.

Mantovani, R. (1999) The molecular biology of the CCAATbinding factor NF-Y. Gene 239, 15-27.

McNabb, D. S., Tseng, K. A. and Guarente, L. (1997) The Saccaromyces cerevisiae Hap5p homologue from fission yeast reveals two conserved domains that are essential for assembly of heterotetrameric CCAAT binding factor. Mol. Cell. Biol. 17, 7008-7018.

Miyoshi, K., Ito, Y., Serizawa, A. and Kurata, N. (2003) OsHAP3 genes regulate chloroplast biogenesis in rice. Plant J. 36, 532540 .
Robson, F., Costa, M. M. R., Hepworth, S. R., Vizir, I., Pineiro, M., Reeves, P. H., Putterill, J. and Coupland, G. (2001) Functional importance of conserved domains in the floweringtime gene CONSTANS demonstrated by analysis of mutant alleles and transgenic plants. Plant J. 28, 619-631.

Romier, C., Cocchiarella, F., Mantovani, R. and Moras, D. (2003) The NF-YB/NF-YC structure gives insight into DNA binding and transcription regulation by CCAAT factor NF-Y. J. Biol. Chem. 278, 1336-1345.

Ruiz-García, L., Maduéno, F., Wilkinson, M., Haughn, G., Salinas, J. and Martínez-Zapater, J., Wenkel, S., Turck, F., Singer, K., Gissot, L., Le Gourrierec, J., Samach, A. and Coupland, G. (2006) CONSTANS and the CCAAT box Binding complex share a functionally important domain and interact to regulate flowering of Arabidopsis. Plant Cell 18, 2971-2984.

Wigge, P. A., Kim, M. C., Jaeger, K. E., Busch, W., Schmid, M., Lohmann, J. U. and Weigel, D. (2005) Integration of spatial and temporal information during floral induction in Arabidopsis. Science 309, 1056-1059.

Xing, Y. Y., Zhang, S. U., Olesen, J. T., Rich, A. and Guarente, L. (1994) Subunit interaction in the CCAAT-binding heteromeric complex is mediated by a very short alpha-helix in HAP2. Proc. Natl. Acad. Sci. USA 91, 3009-3013. 